hep-ph/0412024

UdeA-PE-04/009

\title{
Supernova Neutrinos and the absolute scale of neutrino masses - a Bayesian approach
}

\author{
Enrico Nardi* \\ INFN, Laboratori Nazionali di Frascati, C.P. 13, I00044 Frascati, Italy \\ Instituto de Física, Universidad de Antioquia, A.A.1226, Medellín, Colombia
}

\begin{abstract}
We apply Bayesian methods to the study of the sensitivity to neutrino masses of a Galactic supernova neutrino signal. Our procedure makes use of the full statistics of events and is remarkably independent of astrophysical assumptions. Present detectors can reach a sensitivity down to $m_{\nu} \sim 1 \mathrm{eV}$. Future megaton detectors can yield up to a factor of two improvement; however, they will not be competitive with the next generation of tritium $\beta$-decay and neutrinoless $2 \beta$-decay experiments.
\end{abstract}

\section{Introduction}

It was realized long time ago that neutrinos from a Supernova (SN) can provide valuable informations on the neutrino masses. The basic idea relies on the time-of-flight (tof) delay $\Delta t$ that a neutrino of mass $m_{\nu}$ and energy $E_{\nu}$ traveling a distance $L$ would suffer with respect to a massless particle:

$$
\Delta t=\frac{L}{v}-L \approx 5.1 \mathrm{~ms}\left(\frac{\mathrm{L}}{10 \mathrm{kpc}}\right)\left(\frac{10 \mathrm{MeV}}{\mathrm{E}_{\nu}}\right)^{2}\left(\frac{\mathrm{m}_{\nu}}{1 \mathrm{eV}}\right)^{2} .
$$

Indeed the detection of about two dozens of neutrinos from SN1987 allowed to set model independent upper limits at the level of $m_{\bar{\nu}_{e}}<30 \mathrm{eV}$ [1]. Since SN1987A, several proposal have been put forth to identify the best ways to measure the neutrino tof delays. Often, these approaches rely on the identification of "timing" events that are used as benchmarks for measuring the time lags, as for example the simultaneous emission of gravitational waves 2] or the abrupt interruption of the neutrino flux due to a further collapse into a black hole [3. The less model dependent limits achievable with these methods are at the level of $m_{\nu} \lesssim 3 \mathrm{eV}$, and tighter limits are obtained

${ }^{*}$ Work done in collaboration with J. Zuluaga. Supported in part by COLCIENCIAS. 
only under specific assumptions. A different method to extract informations on the neutrino masses that uses the full statistics of the signal and does not rely on any benchmark event was proposed in Ref. [4] and developed in Ref. [5]. Here we resume the results obtained by applying this method to determine the sensitivity of the SuperKamiokande (SK) water Čerenkov and KAMLAND scintillator detectors, and of the planned experimental facilities Hyper-Kamiokande and LENA.

\section{The method}

In real time detectors, supernova $\bar{\nu}_{e}$ are revealed through to the positrons they produce via charged current interactions, that provides good energy informations as well. Each $\overline{\nu_{e}}$ event corresponds to a pair of energy and time measurements $\left(E_{i}, t_{i}\right)$. In order to extract the maximum of information from a high statistics SN neutrino signal, all the neutrino events have to be used in constructing a suitable statistical distribution, as for example the Likelihood, that can be schematically written as:

$$
\mathcal{L} \equiv \prod_{i} \mathcal{L}_{i}=\prod_{i}\left\{\phi\left(t_{i}\right) \times F\left(E_{i} ; t_{i}\right) \times \sigma\left(E_{i}\right)\right\}
$$

$\mathcal{L}_{i}$ represents the contribution to the Likelihood of a single event with the index $i$ running over the entire set of events, $\sigma(E)$ is the $\bar{\nu}_{e}$ detection cross-section and $F(E ; t)$ is the energy spectrum of the neutrinos, whose time profile can be reconstructed rather accurately directly from the data 4, 5]. The main problem in constructing the Likelihood (2) is represented by the (unknown) time profile of the neutrino flux $\phi(t)$. We construct a flux model by requiring that it satisfies some physical requirements and a criterium of simplicity: $i$ ) the analytical flux function must go to zero at the origin and at infinity; ii) it must contain at least two time scales for the neutrino emission corresponding to the fast rising initial phase of shock-wave breakout and accretion, and the later Kelvin-Helmholtz cooling phase; iii) it must contain the minimum possible number of free parameters. The following model for the flux has all the required behaviors:

$$
\phi(t ; \lambda)=\frac{e^{-\left(t_{a} / t\right)^{n_{a}}}}{\left[1+\left(t / t_{c}\right)^{n_{p}}\right]^{n_{c} / n_{p}}} \begin{cases}\sim e^{-\left(t_{a} / t\right)^{n_{a}}} & (t \rightarrow 0) \\ \sim\left(t_{c} / t\right)^{n_{c}} & (t \rightarrow \infty) .\end{cases}
$$

The five parameters that on the l.h.s of (3) have been collectively denoted with $\lambda$ are: two time scales $t_{a}$ for the initial exponentially fast rising phase and $t_{c}$ for the cooling phase, two exponents $n_{a}$ and $n_{c}$ that control their specific rates and one additional exponent $n_{p}$ that mainly determines the width of the "plateau" between the two phases. Since we are interested only in the neutrino mass squared $m_{\nu}^{2}$, irrespectively of the particular values of the nuisance parameters $\lambda$, starting from the Likelihood (2) we will need to evaluate the marginal posterior probability $p\left(m_{\nu}^{2} \mid D\right)$, that is the probability distribution for $m_{\nu}^{2}$ given the data $D$. This is done by marginalizing the posterior probability with respect to the nuisance parameters:

$$
p\left(m_{\nu}^{2} \mid D, I\right)=N^{-1} \int d \lambda \mathcal{L}\left(D ; m_{\nu}^{2}, \lambda\right) p\left(m_{\nu}^{2}, \lambda \mid I\right) .
$$


$p\left(m_{\nu}^{2}, \lambda \mid I\right)$, that in Bayesian language is called the prior probability of the model, allows us to take into account any available prior information on the parameters $m_{\nu}^{2}$ and $\lambda$. We will use flat priors for all the $\lambda$ 's and, to exclude unphysical values of $m_{\nu}^{2}$, a step function $\Theta\left(m_{\nu}^{2}\right)=1$, (0) for $m_{\nu}^{2} \geq 0,(<0)$.

The dependence on $m_{\nu}^{2}$ could be directly included in the flux (2) by redefining the time variable according to (11). However, it is more convenient to proceed as follows: given a test value for the neutrino mass, first the arrival time of each neutrino is shifted according to its time delay, and then the Likelihood is computed for the whole time-shifted sample. Subtilities in the evaluation of the Likelihood contribution $\mathcal{L}_{i}\left(t_{i}, E_{i}\right)$ arising from the uncertainty $\Delta E_{i}$ in the energy measurement are discussed in Refs. [4, [5].

We have tested the method applying it to a large set of synthetic Monte Carlo (MC) neutrino signals, generated according to two different SN models: SN model I corresponds to the simulation of the core collapse of a $20 M_{\odot}$ star [6] carried out by using the Livermore Group code [8]. In this simulation $\nu_{\mu, \tau}$ opacities were treated in a simplified way, and this resulted in quite large (and probably unrealistic) differences in their average energies with respect to $\bar{\nu}_{e}$. SN model II corresponds to a recent hydrodynamic simulation of a $15 M_{\odot}$ progenitor star [7] carried out with the Garching group code [9]. This simulation includes a more complete treatment of neutrino opacities and results in a quite different picture, since the antineutrino spectra do not differ for more than about 20\%. In both cases the effects of neutrino oscillations in the SN mantle have been properly included in the simulations. Note that the two types of neutrino spectra of SN model I and II fall close to the two extremes of the allowed range of possibilities. This gives us confidence that the results of the method are robust with respect to variations in the spectral characteristics.

\section{Results}

To test the sensitivity of our method, we have analyzed a large number of neutrino samples, grouped into different ensembles of about 40 samples each. For each ensemble we vary in turn the SN model (model I and II), the SN-earth distance $(5,10$, and $15 \mathrm{kpc}$ ) and the detection parameters specific for two operative (SK and KamLAND) and two proposed (Hyper-Kamiokande and LENA) detectors. The results of our recent detailed analysis [5] are summarized in Table 1] In columns 2 and 4 we give the $90 \%$ c.l. upper limits that could be put on $m_{\nu}$ in case its value is too small to produce any observable delay. In columns 3 and 5 we estimate for which value of $m_{\nu}$ the massless neutrino case can be rejected at least in $50 \%$ of the cases. These results confirm the claim 4 that detectors presently in operation can reach a sensitivity of about $1 \mathrm{eV}$, that is seizable better than present results from tritium $\beta$-decay experiments, competitive with the most conservative limits from neutrinoless double $\beta$-decay, less precise but remarkably less dependent from prior assumptions than cosmological measurements. However, in spite of a sizeable improvement, future detectors will not be competitive with the next generation of tritium $\beta$-decay and neutrinoless double $\beta$ decay experiments. 
MODEL 1

MODEL 2

\begin{tabular}{lcccc}
\hline Detector & $\bar{m}_{\text {up }} \pm \Delta m_{\text {up }}$ & $\sqrt{m_{\min }^{2}}$ & $\bar{m}_{\text {up }} \pm \Delta m_{\text {up }}$ & $\sqrt{m_{\min }^{2}}$ \\
\hline a) SK $(10 \mathrm{kpc})$ & $1.0 \pm 0.2$ & 1.0 & $1.1 \pm 0.3$ & 1.2 \\
b) SK $(5 \mathrm{kpc})$ & - & - & $1.1 \pm 0.3$ & 1.0 \\
c) SK $(15 \mathrm{kpc})$ & - & - & $1.6 \pm 0.6$ & 1.4 \\
d) SK+KL $(10 \mathrm{kpc})$ & $1.0 \pm 0.2$ & 0.9 & $1.1 \pm 0.3$ & 1.0 \\
\hline e) HK $(10 \mathrm{kpc})$ & $0.4 \pm 0.1$ & 0.4 & $0.5 \pm 0.1$ & 0.5 \\
f) LENA $(10 \mathrm{kpc})$ & $0.9 \pm 0.2$ & 0.9 & $0.9 \pm 0.3$ & 0.9 \\
\hline
\end{tabular}

Table 1: Results for the fits to $m_{\nu}^{2}$ : a)-c) SK for different SN distances, d) SK plus KamLAND, e) Hyper-Kamiokande, f) LENA. All masses are in eV.

\section{References}

[1] D. N. Schramm, Comments Nucl. Part. Phys. 17, 239 (1987).

[2] D. Fargion, Lett. Nuovo Cim. 31, 499 (1981); N. Arnaud et. al., Phys. Rev. D 65, 033010 (2002).

[3] J. F. Beacom R. N. Boyd and A. Mezzacappa, Phys. Rev. D 63, 073011 (2001); Phys. Rev. Lett. 85, 3568 (2000).

[4] E. Nardi and J. I. Zuluaga, Phys. Rev. D 69, 103002 (2004); E. Nardi, arXiv:astro-ph/0401624.

[5] E. Nardi and J. I. Zuluaga, Submitted to Nucl. Phys. B.

[6] S. E. Woosley et. al., Astrophys. J. 433, 229 (1994).

[7] G. Raffelt et. al., arXiv:astro-ph/0303226, R. Buras, Private Communication.

[8] J. R. Wilson and R. W. Mayle, Phys. Rept. 227, 97 (1993).

[9] M. Rampp and H. T. Janka, Astron. Astrophys. 396, 361 (2002). 